\title{
Magnetization of coupled spin clusters in Ladder Geometry
}

\author{
Emily Chattopadhyay and Indrani Bose
}

21st November 2018

\author{
Physics Department, Bose Institute, 93/1, A.P.C. Road, \\ Calcutta-700009, India
}

\begin{abstract}
In this paper, we construct a class of spin-1/2 antiferromagnetic (AFM) two-chain ladder models consisting of blocks of $n$-spin tetrahedral clusters alternating with two-spin rungs. For $\mathrm{n}=4$ and 6 and in extended parameter regimes, the exact ground state of the ladder is shown to be a product of the ground states of the rungs and the n-spin blocks, in both zero and finite magnetic fields. In the latter case, magnetization/site $m$ versus magenetic field $h$ plot exhibits well-defined magnetization plateaus.
\end{abstract}

PACS numbers: $75.10 \mathrm{Jm}, 75.40 \mathrm{Mg}, 75.50 \mathrm{Ee}$

Spin ladders have been widely studied in recent times as these systems exhibit a variety of novel phenomena in the undoped as well as the doped states ${ }^{1,2}$. There are also several magnetic compounds which can be considered as coupled spin cluster systems in which the dominant exchange interactions occur within clusters of spins. The clusters are coupled through weaker exchange interactions. A prominent example of such systems is that of molecular magnets ${ }^{3}$. Examples of spin clusters are dimers and four-spin plaquettes. The magnetic properties of spin clusters can be determined exactly if the size of the cluster is small. It is of significant interest to find out how the cluster properties are modified in the bulk. In this paper, we propose a class of two-chain spin ladder models which can be defined in terms of $n$-spin clusters and dimers. The specific values of $n$ considered are $n=4$ and 6 though a generalisation to higher $n$ values is also possible. The models describe spins of magnitude $1 / 2$ interacting via modulated antiferromagnetic (AFM) exchange interactions. We show that in a wide parameter regime, the exact ground and low-lying excited states of the full ladder model can be described in terms of the eigenstates of the dimers and n-spin clusters, i.e., the clusters act as decoupled entities even in the bulk. This is also true when the exchange interactions coupling the clusters are of considerable strength. In the same parameter region, the magnetization/site of the ladder in the presence of an external magnetic field exhibits the phenomenon 
of magnetization plateaus. The condition for the appearance of a plateau is given by $^{4}$

$$
S_{u}-m_{u}=\text { integer }
$$

where $S_{u}$ and $m_{u}$ are the total spin and magnetization in unit period of the ground state.

Spin-1/2 ladder models of various types have been extensively studied both in zero and finite magnetic fields ${ }^{5-16}$. In frustrated spin ladder models, extra diagonal exchange couplings (one or two) are present. Hakobyan et al. ${ }^{10}$ have given an overview on the phase diagram of the general frustrated two-chain ladder model. The magnetization process of the general model is, however, yet to be undertaken. Brenig et al. ${ }^{11}$ have introduced a dimerized and completely frustrated two-chain ladder model. The model, in which diagonal exchange interactions are present in every plaquette, is equivalent to a chain of edge sharing tetrahedra. The rung exchange interactions in the ladder model are of strength $J_{2}$. The nearest -neighbour intra-chain and diagonal exchange interactions are of equal strengths and in alternate plaquettes the strengths are $J_{1}$ and $J_{3}$ respectively. Examples of tetrahedral cluster compounds are tellurates of the type $\mathrm{Ca}_{2} \mathrm{Te}_{2} \mathrm{O}_{5} \mathrm{X}_{2}$ with $\mathrm{X}=\mathrm{Cl}, \mathrm{Br} r^{12}$. The class of models we propose describes two-chain spin-ladders with modulated exchange interactions. The spin ladders consist of tetrahedral spin clusters containing $\mathrm{n}$ spins separated by two-spin clusters, i.e., dimers (rungs). In section II, we introduce the models and discuss the magnetization properties in the presence of an external magnetic field. Section III contains a summary and discussion of the major results obtained.

The spin ladder model consists of blocks of tetrahedral clusters containing $\mathrm{n}$ spins separated by rungs of two spins. Fig. 1 . shows the simplest such ladder model with $\mathrm{n}=4$. The tetrahedral clusters are represented by solid lines and the two-spin rungs (dimers) by dashed lines. Within a tetrahedral cluster, the diagonal exchange interactions are of strength $J_{3}$ and the other exchange interactions are of strength $J_{1}$. The rung exchange interaction strengths are $J^{\prime}$ and a rung is coupled to a neighbouring tetrahedral cluster through exchange interactions (dashed lines) of strength $J_{2}$. Periodic boundary condition is assumed to hold true. The spin Hamiltonian describing the ladder model is given by

$$
\begin{gathered}
H=\sum_{i=3 j+1, j=0,1, \cdots}\left[J_{1}\left(\vec{S}_{1 i} \cdot \vec{S}_{1 i+1}+\vec{S}_{2 i} \cdot \vec{S}_{2 i+1}+\vec{S}_{1 i} \cdot \vec{S}_{2 i}+\vec{S}_{1 i+1} \cdot \vec{S}_{2 i+1}\right)\right. \\
\left.+J_{3}\left(\vec{S}_{1 i} \cdot \vec{S}_{2 i+1}+\vec{S}_{2 i} \cdot \vec{S}_{1 i+1}\right)\right]+J^{\prime} \sum_{i=3 j, j=0,1, \cdots} \vec{S}_{1 i} \cdot \vec{S}_{2 i} \\
+J_{2} \sum_{i=3 j+2, j=0,1, \cdots}\left(\vec{S}_{1 i}+\vec{S}_{2 i}+\vec{S}_{1 i+2}+\vec{S}_{2 i+2}\right) \cdot\left(\vec{S}_{1 i+1}+\vec{S}_{2 i+1}\right) \\
=H_{T}+H_{R}+H_{T R}
\end{gathered}
$$


The spin operator $\vec{S}_{1 i}\left(\vec{S}_{2 i}\right)$ is associated with the $i$-th site of the lower (upper) chain of the ladder, the site indices are sequential as shown in Fig. 1. The Hamiltonians $H_{T}$ and $H_{R}$ describe the tetrahedral clusters and the rungs respectively whereas $H_{T R}$ contains the exchange couplings between the tetrahedral clusters and the rungs. The total spin of each rung is a conserved quantity due to the special structure of the Hamiltonian.

We now determine the ground state of the ladder model. Using the method of 'divide and conquer"17, it is easy to show that for $J_{2} \leq \frac{J^{\prime}}{4}$, the exact ground state has all the rungs and the tetrahedral clusters in their ground state spin configurations. A brief sketch of the proof is given in the following. The ground state of a rung is a singlet. The ground state of a tetrahedral spin cluster is a resonating valence bond (RVB) state and has total spin $\mathrm{S}=0$. The ground state is $\left|\psi_{R V B 1}\right\rangle$ for $J_{3}<J_{1}$ and $\left|\psi_{R V B 2}\right\rangle$ for $J_{3}>J_{1}$. The states $\left|\psi_{R V B 1}\right\rangle$ and $\left|\psi_{R V B 2}\right\rangle$ are linear combinations (plus and minus) of two valence bond (VB) states. In one VB state, the two VBs (singlets) are horizontal and in the other vertical.

\section{TABLE I}

\begin{tabular}{|c|c|c|c|}
\hline S & Eigenvalues & $\frac{1}{S^{z}}$ & Eigenstates \\
\hline 0 & $-2 J_{1}+\frac{J_{3}}{2}$ & 0 & $\mid \overline{\left.\psi_{R V B 1}\right\rangle}$ \\
\hline 0 & $-\frac{3 J_{3}}{2}$ & 0 & $\left|\psi_{R V B 2}\right\rangle$ \\
\hline 1 & $-J_{1}+\frac{J_{3}}{2}$ & 0 & $\uparrow \downarrow \downarrow \uparrow-\downarrow \uparrow \uparrow \downarrow$ \\
\hline 1 & $-\frac{J_{3}}{2}$ & 0 & $\uparrow \downarrow \uparrow \downarrow-\downarrow \uparrow \downarrow \uparrow$ \\
\hline 1 & $-\frac{J_{3}}{2}$ & 0 & $\uparrow \uparrow \downarrow \downarrow-\downarrow \downarrow \uparrow \uparrow$ \\
\hline 2 & $J_{1}+\frac{J_{3}}{2}$ & 0 & $\begin{array}{c}\text { } \uparrow \uparrow \downarrow \downarrow+\downarrow \downarrow \uparrow \uparrow+\uparrow \downarrow \uparrow \downarrow+\downarrow \uparrow \downarrow \uparrow \\
+\uparrow \downarrow \downarrow \uparrow+\downarrow \uparrow \uparrow \downarrow \\
\end{array}$ \\
\hline 1 & $-\frac{J_{3}}{2}$ & 1 & $\uparrow \uparrow \downarrow \uparrow \uparrow-\uparrow \downarrow \uparrow \uparrow$ \\
\hline 1 & $-J_{1}+\frac{J_{3}}{2}$ & 1 & $\uparrow \uparrow \uparrow \uparrow \downarrow-\uparrow \uparrow \downarrow \uparrow-\uparrow \downarrow \uparrow \uparrow+\downarrow \uparrow \uparrow \uparrow$ \\
\hline 1 & $J_{1}+\frac{J_{3}}{2}$ & 1 & 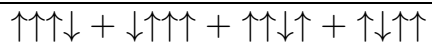 \\
\hline$\overline{2}$ & $J_{1}+\frac{J_{3}}{2}$ & 2 & $\uparrow \uparrow \uparrow \uparrow$ \\
\hline
\end{tabular}

Table I: The energy eigenvalues and eigenvectors of a tetrahedral cluster with exchange interactions of strength $J_{1}$ (horizontal and vertical) and $J_{3}$. The eigenstates $\left|\psi_{R V B 1}\right\rangle$ and $\left|\psi_{R V B 2}\right\rangle$ are the resonating valence bond states.

At $J_{3}=J_{1}$, the ground state of a tetrahedral cluster is doubly degenerate. The two states have a pair of singlets (valence bonds) along either the horizontal or the vertical bonds. The Hamiltonian $H_{T R}$ (Eq. (2)), containing the exchange couplings between the rungs and the tetrahedral clusters, act on the rung singlets to give zero and thus has no contributions to the energy $E_{1}$ of the eigenstate. $E_{1}$ is the sum of the ground state energies of the tetrahedral clusters and the rungs. Let $E_{g}$ be the exact ground state energy of the total Hamiltonian $H$. Then $E_{g}$ is $\leq E_{1}$. Let $\left|\psi_{g}\right\rangle$ be the exact ground state wave function. Then, from variational theory,

$$
E_{g}=\sum_{i}\left\langle\psi_{g}\left|H_{i}\right| \psi_{g}\right\rangle+\sum_{i}\left\langle\psi_{g}\left|H_{i}^{\prime}\right| \psi_{g}\right\rangle \geq \sum_{i}\left(E_{i o}+E_{i o}^{\prime}\right)
$$




$$
H=\sum_{i}\left(H_{i}+H_{i}^{\prime}\right)
$$

where $H_{i}$ 's are the tetrahedral cluster Hamiltonians with the ground state energy $E_{i o}$ (Table I) and $H_{i}^{\prime}$ 's are the six spin cluster Hamiltonians, each of which contains the rung exchange interaction Hamiltonian and the eight exchange couplings (four horizontal and four diagonal) which connect the rung to nearestneighbour tetrahedral clusters. The ground state energy of $H_{i}^{\prime}$ is $E_{i o}^{\prime}$. For $J_{2} \leq \frac{J^{\prime}}{4}, E_{i o}^{\prime}$ is the energy of a singlet across the rung. We can now write down the inequality,

$$
\sum_{i}\left(E_{i o}+E_{i o}^{\prime}\right) \leq E_{g} \leq E_{1}
$$

$E_{1}$ is, however, exactly equal to $\sum_{i}\left(E_{i o}+E_{i o}^{\prime}\right)$ since it is the sum over the ground state energies of all the rungs and the tetrahedral clusters. Thus, $E_{g}=E_{1}$, i.e., the exact eigenstate is also the exact ground state of the full ladder model. The ground state has the novel structure of islands of four-spin RVB configurations in the tetrahedral clusters separated by singlet spin configurations along the rungs. The exact ground state energy is $E_{g}=N\left(E_{i 0}-3 \frac{J^{\prime}}{4}\right)$ where $\mathrm{N}$ is the total number of tetrahedral clusters as well as rungs in the ladder. $E_{i 0}=-2 J_{1}+\frac{J_{3}}{2}$ for $J_{3}<J_{1}$ and $E_{i 0}=-\frac{3 J_{3}}{2}$ for $J_{3}>J_{1}$ (Table I). When $J_{3}=J_{1}$, the exact ground state is highly degenerate. The number of such states is $2^{N}$.

We now want to check whether the exact ground state is still a product of the ground states of the rungs and the tetrahedral clusters when $J_{2}$ is made larger than $\frac{J^{\prime}}{4}$. For this, the total Hamiltonian $H$ (Eq. (2)) is written as a sum over six-spin sub-Hamiltonians, $h_{i}$ 's, i.e., $H=\sum_{i} h_{i}$. Each sub-hamiltonian describes a tetrahedral cluster coupled to a rung. The six-spin sub-Hamiltonian can be diagonalised exactly to obtain the ground state energy. Again, one uses the method of 'divide and conquer'. When the six-spin sub-Hamiltonians are added together to obtain the full Hamiltonian, the $J_{1}, J_{3}, J^{\prime}$ bonds are counted twice and the $J_{2}$ bonds only once. One can identify the region of parameter space in which the exact ground state of the full ladder is of the product form. Fig. 2 shows the phase boundaries, in the parameter space of $\frac{J_{2}}{J_{1}}$ and $\frac{J^{\prime}}{J_{1}}$ for different values of $\frac{J_{3}}{J_{1}}$. In the parameter regime below each phase boundary, the exact ground state is a product over the ground states of the rungs and the tetrahedral clusters. One finds that in certain parameter regimes $J_{2}$ can be larger than $\frac{J^{\prime}}{4}$ and the exact ground state continues to be of the product form.

We next include an external magnetic field term $-h \sum_{i=1}^{6 N} S_{i}^{z}$ in the Hamiltonian $H$ (Eq. (2)), where $6 \mathrm{~N}$ is the total number of sites in the ladder. We first consider the case of a single tetrahedral cluster in the presence of a magnetic field. The magnetic field couples to the z-component of the total spin of the cluster, $S_{t o t}^{z}$, which is a conserved quantity. The ground state energy $E_{g}\left(S_{t o t}^{z}\right)$ at $h=0$ for $S_{t o t}^{z}=0,1$ and 2 can be obtained from Table I. When the external field $h \neq 0$, the ground state in each $S_{\text {tot }}^{z}$ subspace is $E_{g}\left(S_{t o t}^{z}, h\right)=E_{g}\left(S_{\text {tot }}^{z}, 0\right)-h S_{\text {tot }}^{z}$. 
The ground state magnetization curve can be easily obtained. Consider the case $J_{3}<J_{1}$. The magnetization per site $m$ is zero from $h=0$ upto a critical field $h_{c_{1}}=J_{1}$. For $h_{c_{1}}<h<h_{c_{2}}=2 J_{1}, m=\frac{1}{4}$ and beyond $h=h_{c_{2}}$, the saturation magnetization, $m=\frac{1}{2}$, is obtained. Thus there are three magnetization plateaus at $m=0, \frac{1}{4}$ and $\frac{1}{2}$. For the external field $h=0$, we have already seen that there is an extended parameter regime in which the exact ground state of the full ladder is a product of the ground states of the rungs and the tetrahedral clusters. We now investigate whether the same holds true for a finite magnetic field. Again, one uses the method of 'divide and conquer' and the sub-Hamiltonian used is a six-spin cluster consisting of a tetrahedral cluster and a rung. For the full ladder, one can identify a region (region $\mathrm{A}$ ) in parameter space in which for $0<h<h_{c_{1}}, m$ is zero. At $h_{c_{1}}$, there is a jump in the value of $m$ to $m=\frac{1}{6}$ and a plateau is obtained for $h$ upto $h_{c_{2}}$ (Fig. 3). When $h_{c_{1}}<h<h_{c_{2}}$, the exact ground state has the tetrahedral clusters in their $S^{z}=1$ ground states and the rungs in singlet spin configurations. Since, the number of tetrahedral clusters is $\mathrm{N}$ and the total number of sites is $6 \mathrm{~N}$, the magnetization/site $m$ in the ground state is $\frac{1}{6}$. The quantization condition in Eq. (1) is obeyed as unit period of the ground state contains six spins so that $S_{u}=3$ and the magnetization $m_{u}$ in the unit period is 1 . At $h_{c_{2}}$, there is a second jump in $m$ from $\frac{1}{6}$ to $\frac{1}{3}$. When $h_{c_{2}}<h<h_{c_{3}}$, the exact ground state has the tetrahedral clusters in their $S^{z}=2$ ground states and the rungs in singlet spin configurations. In this case, $S_{u}$ and $m_{u}$ in Eq. (1) are 3 and 2 respectively. At $h=h_{c_{3}}$, there is a jump in $m$ from $\frac{1}{3}$ to the saturation magnetization $\frac{1}{2}$. For $J_{3}<J_{1}, h_{c_{1}}, h_{c_{2}}$ and $h_{c_{3}}$ have the values $J_{1}, 2 J_{1}$ and $J^{\prime}+J_{2},\left(2 J_{1}<\left(J^{\prime}+J_{2}\right)\right)$ respectively. There are other parameter regions (regions $\mathrm{B}$ and $\mathrm{C}$ ) in the parameter space in which the full plateau structure in the $m$ versus $h$ plot, as shown in Fig. 3, is not obtained. Fig. 4 shows the phase diagram for the full ladder in a magnetic field in the $\frac{J^{\prime}}{J_{1}}$ vs. $\frac{J_{3}}{J_{1}}$ parameter space and for $\frac{J_{2}}{J_{1}}=0.2$. The region A exhibits the full plateau structure in $m$ vs. $h$ as shown in Fig. 3. In region B, the jump in $m$ from 0 to $\frac{1}{6}$ occurs at $h=h_{c_{1}}$ (Fig. 3) but beyond $h_{c_{2}}$, the ground state is no longer of the product form. In region $\mathrm{C}$, the ground state loses its simple product structure beyond $h=h_{c_{1}}$. Similar phase diagrams are obtained for higher values of $\frac{J_{2}}{J_{1}}$ and also for $J_{3}>J_{1}$. One can generalise the ladder model shown in Fig. 1 by assigning different coupling strengths $J_{1}, J_{4}$ and $J_{3}$ to the vertical, horizontal and diagonal couplings of the tetrahedral clusters. Again, results similar to the case $J_{1}=J_{4}$ are obtained. With $J_{4}=J_{3}=J_{2}$ and $J_{1}=J^{\prime}$, the two-chain frustrated ladder model introduced by Bose and Gayen ${ }^{18}$ is recovered. In a finite magnetic field $h$, the magnetization/site $m$ vs. $h$ has a simple plateau structure ${ }^{6}$.

Another generalisation of the ladder model shown in Fig. 1 is to replace a tetrahedral cluster by a block of tetrahedral clusters. Fig. 5 shows an example in which the block contains two tetrahedral clusters. The six-spin blocks are separated by two-spin rungs. Again, one can show that in an extended parameter regime, the ground state has the product form in both zero and finite magnetic fields. The exact ground state is the product of the ground states of the six-spin blocks and the rungs. The ground state of a six-spin block is a RVB state. An 
extra magnetization plateau exists for $h_{c_{3}}<h<h_{c_{4}}$ in which the ground state has all the six-spin blocks in their $S^{z}=3$ ground state configurations and the rungs are in singlet spin configurations. At $h=h_{c_{4}}, m$ jumps to its full saturation value. Fig. 6 is the phase diagram similar to Fig. 4 for the full ladder with $\frac{J_{2}}{J_{1}}=0.2$. In the 'divide and conquer' method, the full ladder Hamiltonian is a sum over eight-spin sub-Hamiltonians. Each sub-Hamiltonian describes the interactions in a block of spins consisting of two tetrahedral clusters and a single rung. In region $A_{1}$, the full plateau structure in $m$ vs. $h$ is obtained. In regions $B_{1}, C_{1}$ and $D_{1}$, the ground state no longer has the product form beyond the fields $h_{c_{3}}, h_{c_{2}}$ and $h_{c_{1}}$ respectively. Similar phase diagrams are obtained for higher values of $\frac{J_{2}}{J_{1}}$. One can generalise the ladder models shown in Figs. 1 and 6 by making the blocks of tetrahedral clusters of bigger size (the total number of spins in a block may be $4,6,8,10 \ldots$. etc.). Two-spin rungs separate the blocks of spins. In certain parameter regimes, the exact ground state is possibly the product of the exact ground states of the rungs and the blocks of tetrahedral clusters. A full study of such ladder models is yet to be undertaken.

In this paper, we have described a class of two-chain ladder models consisting of blocks of tetrahedral clusters, containing $\mathrm{n}$ spins, separated by two-spin rungs. We have specifically considered two cases: $n=4$ and 6 . We have shown that in an extended parameter regime, the ground state of the ladder is a product over the ground states of the rungs and the blocks of tetrahedral clusters. For $n=4$, we have shown that the exact ground state consists of RVB spin configurations in the tetrahedral clusters and the rungs are in singlet spin configurations. For $J_{3}=J_{1}$, the ground state is highly degenerate. When $J_{3}$ is $<J_{1}\left(>J_{1}\right)$, the tetrahedral cluster is in the RVB state $\left|\psi_{R V B 1}\right\rangle\left(\left|\psi_{R V B 2}\right\rangle\right)$ and the exact ground state of the full ladder model is non-degenerate. A notable feature of the ladder model is the presence of singlet excitations in the triplet spin gap in certain parameter regimes. As already pointed out in earlier references ${ }^{11,12}$, the singlet energy level $\left|\psi_{R V B 2}\right\rangle$ of a tetrahedral cluster crosses the triplet energy level at $J_{3}=\frac{J_{1}}{2}$ (Table I). Thus for $\frac{J_{1}}{2}<J_{3}<J_{1}$, the singlet excitation described by $\left|\psi_{R V B 2}\right\rangle$ falls in the triplet gap. Similarly, for $J_{3}>J_{1},\left|\psi_{R V B 2}\right\rangle$ is the ground state and for $J_{1}<J_{3}<2 J_{1}$, the singlet excitation corresponding to $\left|\psi_{R V B 1}\right\rangle$ falls in the triplet gap. These features carry over to the case of the full ladder model in the parameter region in which the exact ground state can be written in a product form. The existence of singlet excitations in the triplet spin gap is a characteristic feature of some other AFM spin systems which include the $S=\frac{1}{2}$ Heisenberg antiferromagnet (HAFM) on the kagomé lattice ${ }^{19}$, the $S=\frac{1}{2}$ HAFM on the pyrochlore lattice ${ }^{20}$ and some $S=\frac{1}{2}$ AFM spin models on the $\frac{1}{5}$-depleted square lattice ${ }^{21,22}$.

The model shown in Fig. 1 can be generalised to bigger blocks of tetrahedral clusters. (Fig. 5 shows blocks of two tetrahedral clusters). Instead of the tetrahedral cluster shown in Fig. 1, one can also consider a generalised tetrahedral cluster with the horizontal, vertical and diagonal exchange interactions of different strengths. Again, in an extended parameter regime, the exact ground state is found to be of the product form. The ground states have the interesting structure of islands of RVB spin configurations separated by singlet spin con- 
figurations along the rungs. This type of exact ground state is not known for other spin models including ladders with modulated exchange interactions.

The ladder models have also been studied in an external magnetic field $h$. In the parameter regime in which the ground states in the different magnetization subspaces are of the product form, the magnetisation/site $m$ as a function of $\mathrm{h}$ exhibits plateaus (Fig. 3). The quantization condition in Eq. (1) is obeyed at each plateau. Figs. 4 and 6 show the phase diagrams for the ladder models of the types shown in Figs. 1 and 5. Both the phase diagrams show that there are extended regions in parameter space in which the ground states in different magnetization subspaces are of the product form. Kolezhuk ${ }^{23}$ has studied magnetization plateaus in a spin system consisting of strongly coupled dimers which are again weakly coupled in a planar arrangement of zigzag interactions. In our ladder models, we have two different type of clusters: dimers (two-spin rungs) and tetrahedral clusters. Further studies are needed to obtain the phase diagrams of the ladder models in the full parameter space.

The authors thank S. Ramasesha and K. Tandon for letting them use their Heisenberg Calculator (exact diagonalisation program) for quantum spin systems. E. Chattopadhyay is supported by the Council of Scientific and Industrial Research, India under sanction No. 9/15(186)/97-EMR-I. 


\section{References}

[1] E. Dagotto and T. M. Rice, Science 271, 618 (1996)

[2] E. Dagotto, Rep. Prog. Phys. 62, 1525 (1999)

[3] O. Kahn, Molecular Magnetsm (VCH Publisher, New York 1993)

[4] M. Oshikawa, M. Yamanaka and I. Affleck, Phys. Rev. Lett. 78, 1984 (1997)

[5] D. C. Cabra, A. Honecker and P. Pujol, Phys. Rev. Lett. 79, 5126 (1997); Phys. Rev.B 58, 6241 (1998)

[6] K. Tandon, S. Lal, S. K. Pati, S. Ramasesha and D. Sen, Phys. Rev. B 59, $396(1999)$

[7] F. Mila, Eur. Phys. J. B 6, 201 (1998)

[8] D. C. Cabra, M. D. Grynberg, Phys. Rev. Lett. 82, 1768 (1999)

[9] D. C. Cabra, M. D. Grynberg, A. Honecker and P. Pujol, cond-mat/0010376 and references therein.

[10] T. Hakobyan, J. H. Hetherington and M. Roger, cond-mat/0011355

[11] W. Brenig, K. W. Becker and P. Lemmens, cond-mat/0101460; W. Brenig and K. W. Becker, cond-mat/0105096

[12] M. Johnsson, K. W. Törnross, F. Mila and P. Millet, Chem. Mater. 12, $2853(2000)$

[13] A. Honeker, F. Mila, M. Troyer, Eur. Phys. J. B15 227 (2000)

[14] E. Müller-Hartmann, Rajiv R.P.Singh, C. Knetter, G.S. Uhrig, Phys. Rev. Lett. 841808 (2000)

[15] A. Koga, K.Okunishi, N. Kawakami, Phys. Rev B 625558 (2000)

[16] J. Schulenburg and J. Richter, cond-mat/0107137

[17] P. W. Anderson, Phys. Rev. 83, 1260 (1951)

[18] I. Bose and S. Gayen, Phys. Rev. B 48, 10653 (1993); Y. Xian, Phys. Rev. B 52, 12485 (1995)

[19] C. Waldtmann et al., Eur. Phys. J. B 2, 501 (1998)

[20] B. Canals and C. Lacroix, Phys. Rev. Lett. 80, 2933 (1998)

[21] I. Bose and A. Ghosh, Phys. Rev. B 56, 3154 (1997)

[22] M. Albrecht, F. Mila and D. Poilblanc, Phys. Rev. B 54, 5856 (1996)

[23] A. K. Kolezhuk, Phys. Rev. B 59, 4181 (1999) 


\section{Figure Captions}

Fig. 1. Two-chain ladder model consisting of tetrahedral clusters (solid lines) coupled to two-spin rungs (dashed lines). The exchange interaction strengths are as shown in the Figure.

Fig. 2. Phase diagram of the ladder model (Fig.1) in the parameter space of $\frac{J_{2}}{J_{1}}$ and $\frac{J^{\prime}}{J_{1}}$. The parameter space below a solid line corresponds to the phase in which the exact ground state is a product over the ground states of the rungs and the tetrahedral clusters.

Fig. 3. Plot of magnetization/site $m$ versus external magnetic field $h$ for the two-chain ladder model shown in Fig. 1. The plot is obtained in the parameter region in which the exact ground states in different $S_{t o t}^{z}$ subspaces have the product form. Two non-trivial magnetization plateaus occur at $m=\frac{1}{6}$ and $m=\frac{1}{3}$.

Fig. 4. Phase diagram of the ladder model (Fig. 1) in a finite magnetic field and in the parameter space of $\frac{J^{\prime}}{J_{1}}$ and $\frac{J_{3}}{J_{1}}$ with $\frac{J_{2}}{J_{1}}=0.2$. The regions A, B and $\mathrm{C}$ are explained in the text.

Fig. 5. A two-chain spin ladder which consists of blocks of two tetrahedral clusters coupled to two-spin rungs (dashed lines). The exchange interaction strengths are as shown in the Figure.

Fig. 6. Phase diagram of the ladder model (Fig. 5) in a finite magnetic field and in the parameter space of $\frac{J^{\prime}}{J_{1}}$ and $\frac{J_{3}}{J_{1}}$ with $\frac{J_{2}}{J_{1}}=0.2$. The regions $A_{1}, B_{1}$, $C_{1}$ and $D_{1}$ are explained in the text. 


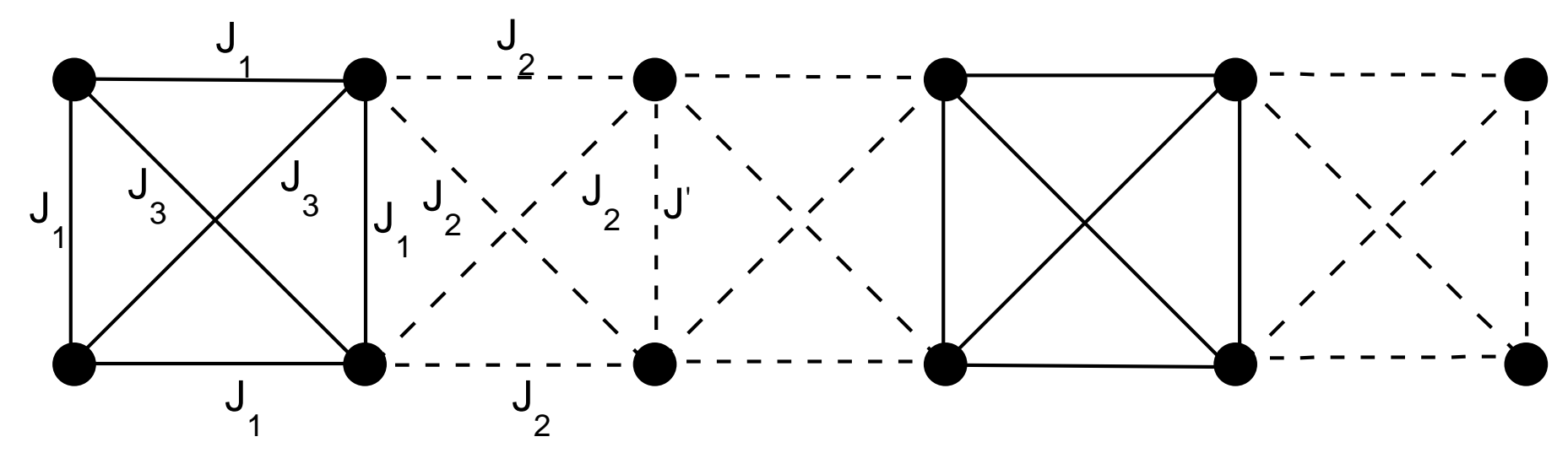




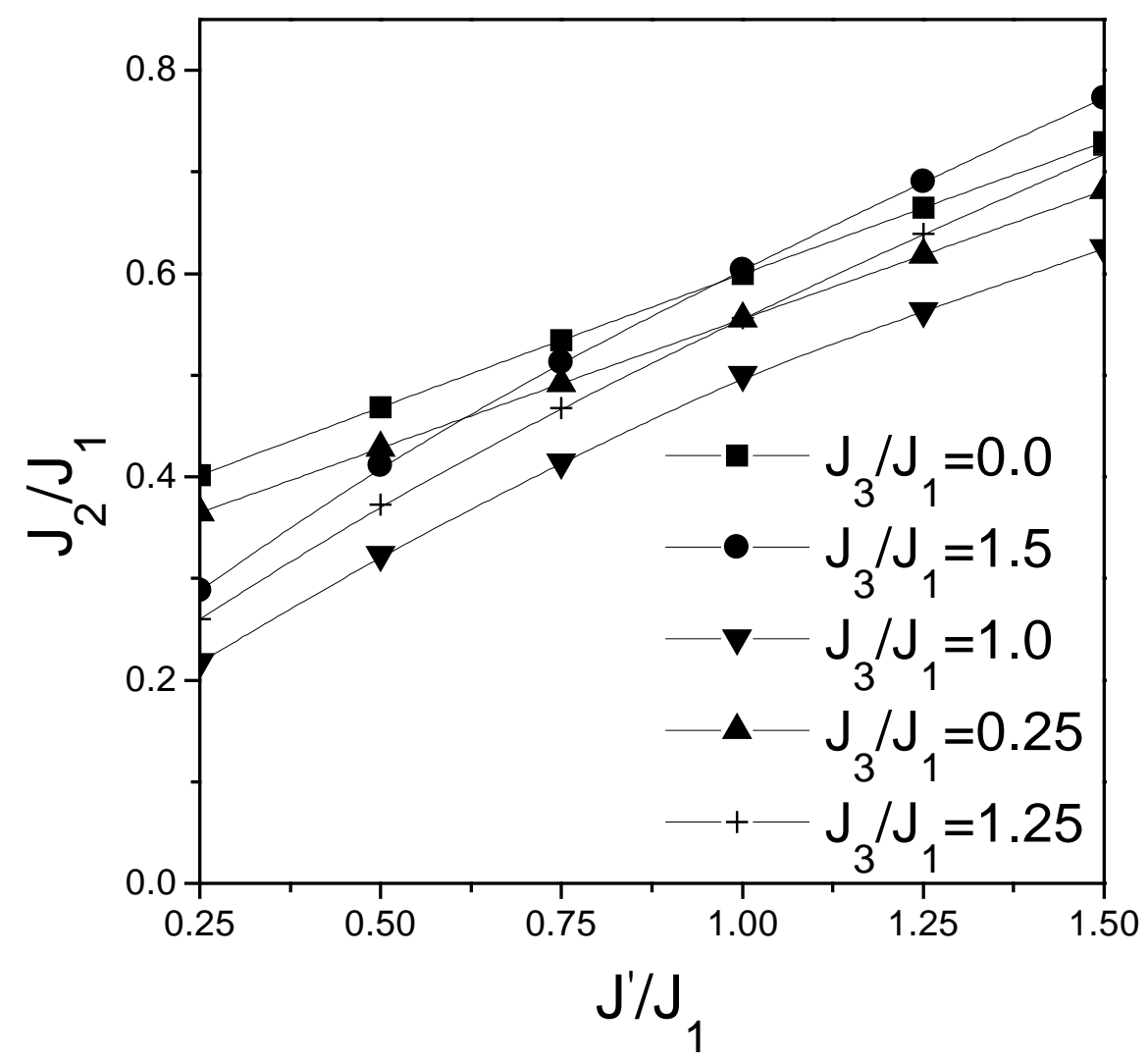




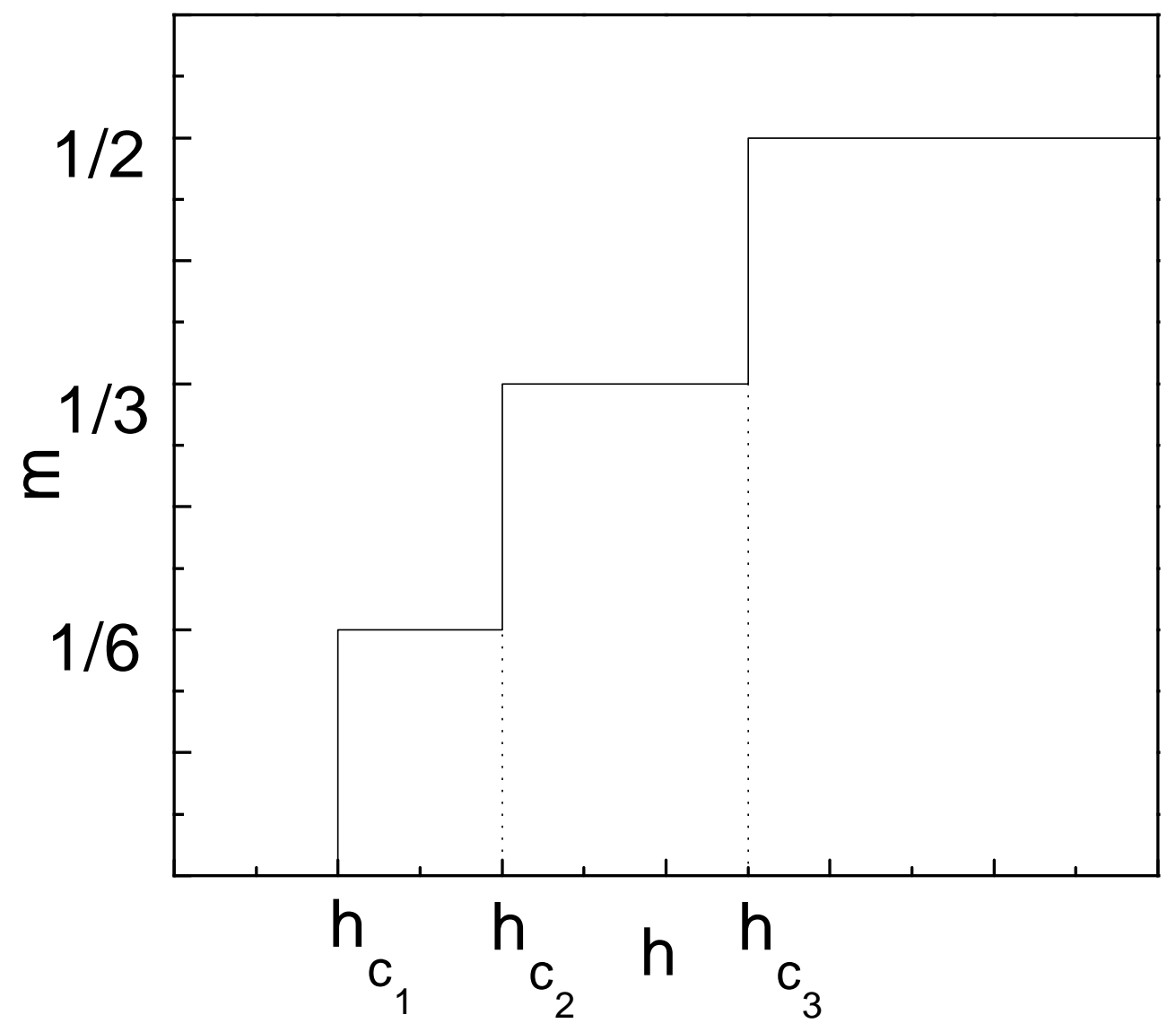




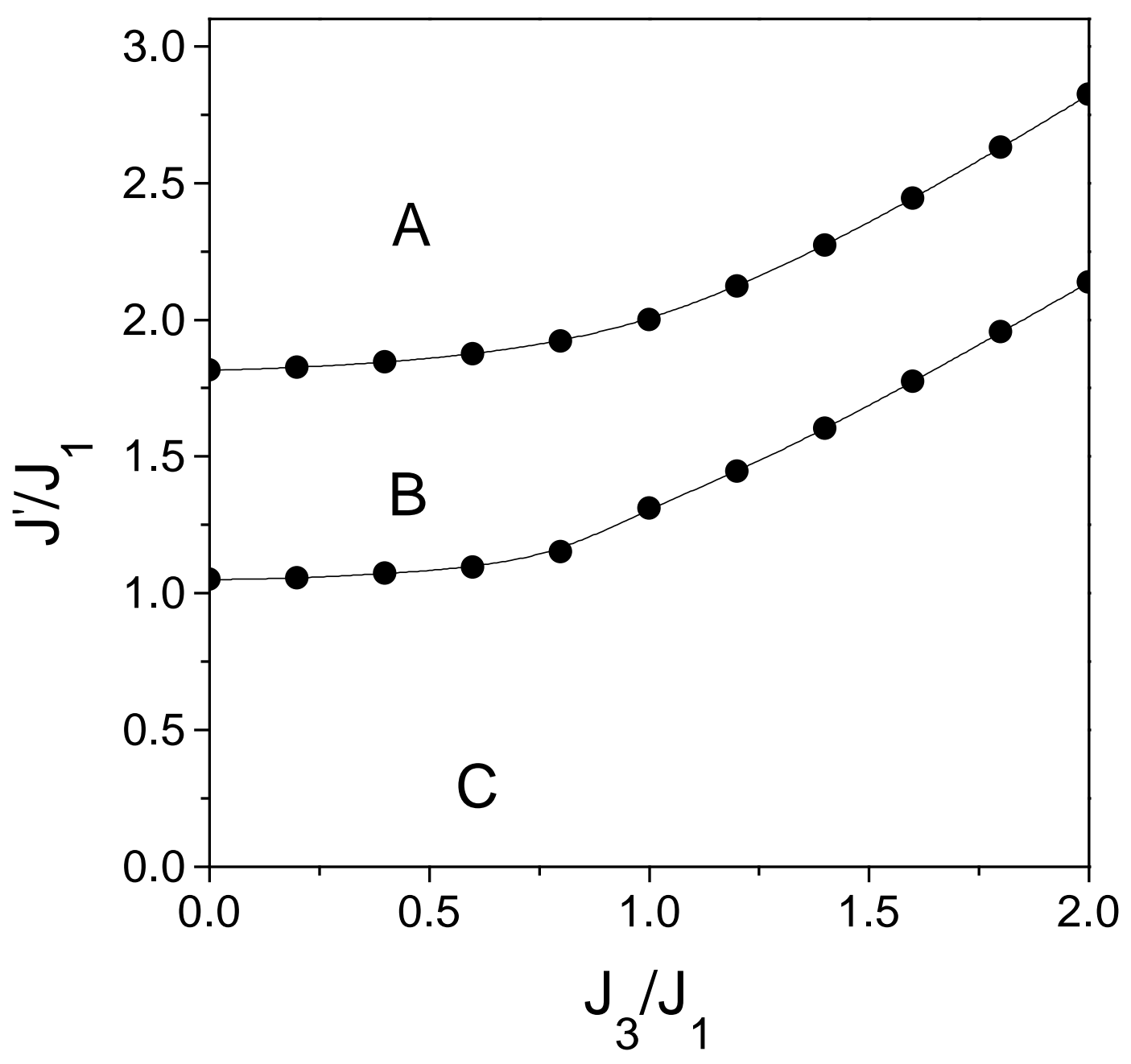




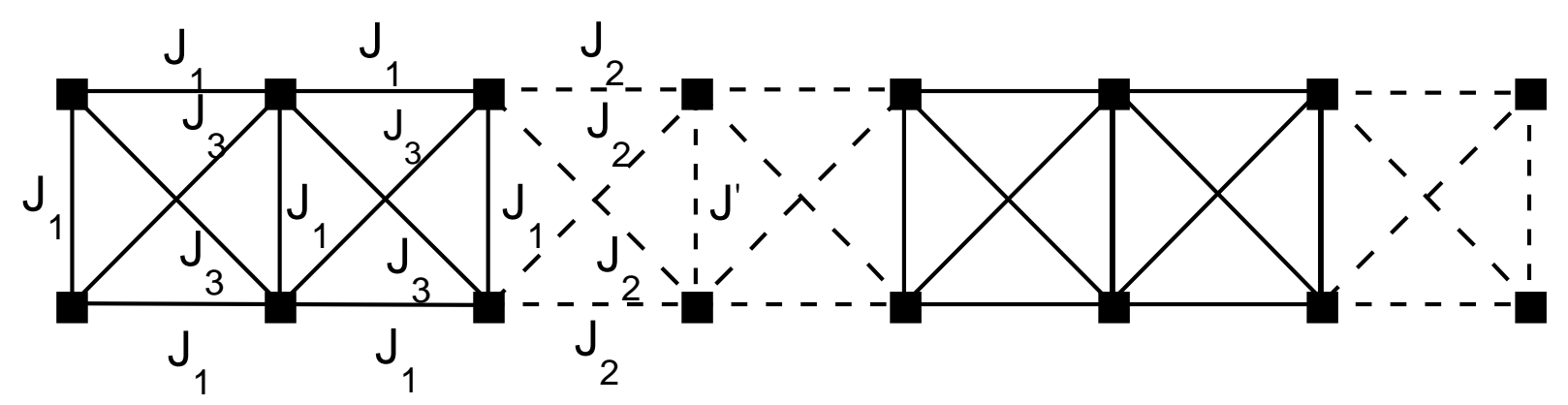




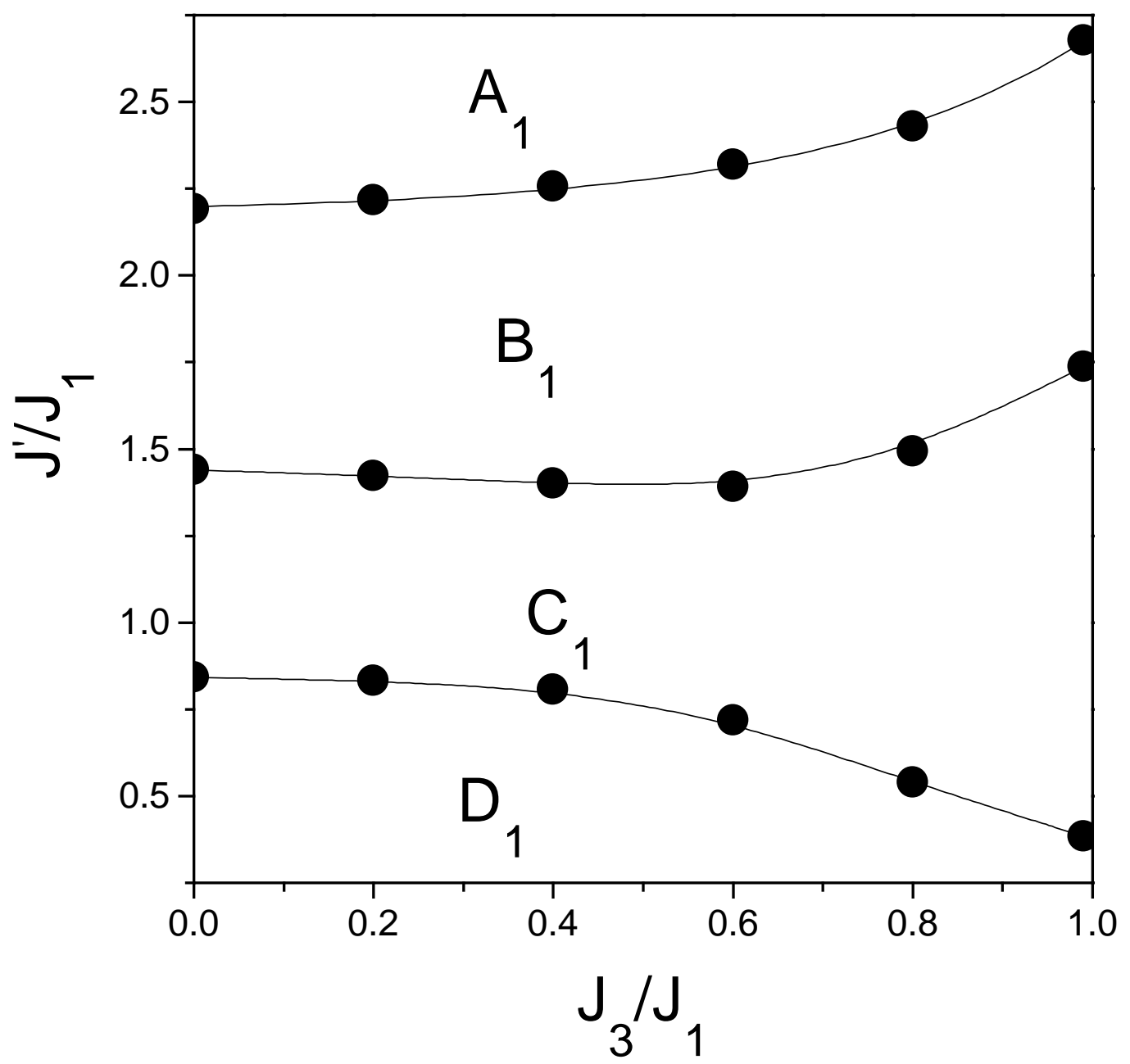

